\title{
Perceived acceptability of progesterone to prevent preterm births and low birth weight among HIV-infected and HIV-uninfected Zambian pregnant women
}

\author{
This article was published in the following Dove Press journal: \\ International Journal of Women's Health \\ 16 November 2017 \\ Number of times this article has been viewed
}

\author{
Jeffrey Man Hay Wong' \\ Neha Kanga' \\ Nupur Dogra' \\ Mary Shilalukey Ngoma ${ }^{2}$ \\ Lena Serghides ${ }^{3}$ \\ Michael Silverman ${ }^{4}$ \\ 'Faculty of Medicine, University \\ of Toronto, Toronto, ON, Canada; \\ ${ }^{2}$ Department of Pediatrics, University \\ of Zambia, Lusaka, Zambia; ${ }^{3}$ Toronto \\ General Research Institute, University \\ Health Network, Toronto, ON, \\ Canada; ${ }^{4}$ Department of Infectious \\ Diseases, Western University, London, \\ ON, Canada
}

Introduction: Intramuscular and vaginal progesterone are recommended for prevention of preterm labor (PTL) in women with risk factors. Studies are emerging to indicate that HIVinfected women on combination antiretroviral therapy (cART) are at risk of PTL and low birth weight (LBW), and may benefit from supplemental progesterone. This study aims to determine the perceived acceptability of various modes of progesterone supplementation to prevent PTL and LBW in HIV-infected and HIV-uninfected women.

Methods: HIV-infected and HIV-uninfected women were recruited in Lusaka, Zambia. The participants completed a questionnaire to assess their willingness to take oral, vaginal, or intramuscular progesterone supplementation for preventing PTL and LBW, preferred modes of supplementation, and concern for PTL and LBW.

Results: The study questionnaire was completed by 147 participants. Of the participants, $98.6 \%$ would consider using a medication to help prevent PTL and LBW, of whom 97.9\% would consider using an oral form of progesterone. In addition, $83.3 \%$ and $84.0 \%$ of women would consider intramuscular and vaginal (gel or tablet) administration of progesterone respectively. Between intramuscular and vaginal modes of progesterone, $60.5 \%$ of participants $(n=147)$ preferred intramuscular progesterone, while $39.5 \%$ preferred vaginal progesterone. There was no difference in preference between HIV-infected $(n=70)$ and HIV-uninfected $(n=77)$ women. Conclusions/implications: Pregnant Zambian women demonstrated a high degree of acceptance for all modes of progesterone supplementation for the prevention of PTL and LBW. Women preferred intramuscular over vaginal supplementation. Progesterone supplementation can be considered a feasible intervention for preventing PTL and LBW in both HIV-infected and HIV-uninfected pregnant Zambian women.

Keywords: HIV/AIDS, progesterone, preterm labor, low birth weight, global health

\section{Plain language summary}

The supplementation of a hormone called progesterone through intramuscular injections or vaginal administration is well accepted as a method of preventing preterm labor in individuals with specific risk factors. These risk factors include previous preterm births and having a short cervix on ultrasound. There is also emerging evidence suggesting pregnant women on HIV medications may benefit from progesterone supplementation. While this is an accepted intervention, there appears to be an uptake gap in the practice of progesterone supplementation in developing nations. Our study surveyed 147 pregnant Zambian women to understand whether they would find progesterone supplementation acceptable. Our study demonstrated that $94.5 \%$ of Zambia women felt that intramuscular or vaginal progesterone supplementations are
Correspondence: Jeffrey Man Hay Wong Department of Infectious Diseases

Western University London, 268

Grosvenor St B3-414, London,

ON N6A4V2, Canada

Tel +l 2892421598

Email jeffreymh.wong@mail.utoronto.ca
International Journal of Women's Health 2017:9 827-833

827 
acceptable interventions for preventing preterm labor. In our sample, $60 \%$ of women would prefer intramuscular injections over vaginal administration. This is important data to allow us to feasibly move forward with implementing progesterone supplementation programs in Zambia and other similar developing countries.

\section{Introduction}

Preterm labor (PTL) is a major contributor to neonatal mortality and morbidity worldwide, ${ }^{1}$ with low-income countries having disproportionately greater rates ${ }^{2}$ and more limited resources to manage complications of PTL. ${ }^{3}$ Many risk factors have been reported for PTL including maternal age, previous PTL, cervical insufficiency, multiple gestations, comorbid maternal conditions (eg, hypertension, diabetes, anemia), and infections. . $^{1,4,5}$

In individuals at increased risk of PTL, supplemental progesterone is considered a preventative strategy. ${ }^{6,7}$ The American Committee of Obstetricians and Gynecologists $(\mathrm{ACOG})^{8}$ and the Society of Obstetricians and Gynecologists of Canada (SOGC) ${ }^{9}$ guidelines identify previous PTL and short cervix as indications to start supplemental progesterone. Both intramuscular and vaginal preparations of progesterone are acceptable for preventing PTL; there is currently no consensus on either's superiority. 8,9

Pooled analyses and meta-analyses have also demonstrated an association between the use of combination antiretroviral therapy (cART) and PTL and low birth weight (LBW). ${ }^{10,11}$ Protease inhibitors in particular have been associated with increased risk of PTL. ${ }^{12}$ Papp et $\mathrm{al}^{13}$ identified an association between protease inhibitor (PI)-based cART and lower progesterone levels. In both humans and mice, lower progesterone levels were correlated with lower fetal/birth weight; and antenatal supplemental progesterone improved fetal weights in pregnant mice. ${ }^{13}$ These data provide a rationale for progesterone supplementation in pregnant women on PI-based cART.

Existing literature on progesterone acceptability is limited to contraception ${ }^{14,15}$ and luteal support, ${ }^{16}$ and generally demonstrate a high degree of acceptability. In a study based in Kenya, Nigeria, and Senegal, ${ }^{14} 98 \%$ of women reported satisfaction after 6-month use of a progesterone vaginal ring used for contraceptive benefits. One study ${ }^{16}$ on luteal support found greater patient satisfaction with vaginal over intramuscular progesterone. In a small qualitative Malawian study of four mothers, ${ }^{17}$ all were open to intramuscular or vaginal progesterone supplementation to prevent PTL; vaginal administration was preferred due to perceived costs with weekly injections. The study also identified a large knowledge gap regarding the use of supplemental progesterone in the prevention of PTL.
The primary objective of our study was to identify the perceived acceptability of oral, intramuscular, and vaginal progesterone supplementation for preventing PTL and LBW in HIV-infected and HIV-uninfected pregnant Zambian women. The secondary objectives were to identify the level of concern for PTL and LBW in pregnant Zambian women, and the factors that influence the acceptability of progesterone supplementation.

\section{Methods \\ Study design}

The data on acceptability of progesterone supplementation were collected as part of the Progesterone Study in Lusaka, Zambia. The primary aim of the Progesterone Study is to assess the association between maternal serum progesterone levels and birth outcomes in two cohorts of pregnant women: HIV-infected pregnant women on cART, and HIV-uninfected pregnant women. Research ethics board review and approval for this study were received from the University of Zambia in Lusaka, Zambia and from the University Health Network in Toronto, Canada.

All participants provided written informed consent for this study. Data collection from enrolled participants occurred at gestational week (GW) 24-28, GW 35-37, and postpartum. The Baseline Questionnaire (Appendix 1) was administered at GW 24-28, and captured participant data on demographics, past medical and obstetrical history, and social history. At GW 35-37, the Supplemental Progesterone Questionnaire (Appendix 2) captured perception of risk of adverse birth outcomes, past experience with progesterone, and future willingness to take oral, vaginal, or intramuscular progesterone supplementation in the context of preventing future adverse birth outcomes.

\section{Recruitment}

Women were recruited in partnership with the Antenatal Care Program from the Chelstone District Health Centre in Lusaka, Zambia. Using convenience sampling, women who arrived at the health center for prenatal visits were screened by nurses and/or midwives for eligibility. Eligible women were referred to the study nurse for further screening and recruitment. Women were compensated $40 \mathrm{ZMK}$ ( 6 USD) at each visit.

Inclusion criteria: $>18$ years of age, pregnant and $<28$ weeks gestation, capacity for informed consent, completed HIV testing during pregnancy, willingness to follow-up, and if HIV-positive having a known list of cART medications. Exclusion criteria: current use of supplemental progesterone, on treatment for active tuberculosis or malaria, or on protease inhibitor based cART. 


\section{Data collection}

Participant demographics were drawn from responses to the Baseline Questionnaire. The acceptability of progesterone supplementation was assessed using responses to the Supplemental Progesterone Questionnaire, administered at the first follow-up visit. In the event of a premature delivery or a missed visit, the questionnaire was administered postpartum.

All questionnaires were administered by the study nurse in English, Nyanja, or Bemba.

\section{Statistical analysis}

Sample size calculations were performed for the parent study. The parent study aimed to accrue a sample size of 60 per group to detect a difference of $25 \%$ in progesterone levels (based on our in vitro and in vivo studies) between HIV-infected and HIV-uninfected participants with a power of 0.80 and an alpha of 0.05 . Categorical variables were analyzed with the Chi square test or Fisher's exact test. Continuous variables were analyzed using Student's $t$-test or Mann-Whitney test as appropriate. A $p$-value of $<0.05$ was used as the cutoff for two-sided significance. Statistical analysis was completed on SPSS Statistics Version 21.0 (IBM Corporation, Armonk, NY, USA).

\section{Results}

A total of 226 participants were recruited into the study at GW 24-28, of whom 147 presented for a follow-up visit (70 HIV-infected, 77 HIV-uninfected women). Fourteen women presented outside the designated time frame of GW 35-37. Participants lost to follow-up were more likely to be married than those retained in the study; the two groups were otherwise similar.

The demographic data are presented in Table 1. HIVinfected women were older ( 29.9 vs 26.7 years; $p=0.001$ ) and had a lower education status compared to HIV-uninfected women. Gravidity and parity were also higher in the HIVinfected compared to the HIV-uninfected group (median [range] for gravidity: $3[1,8]$ vs $2[1,7] ; p=0.016$; for parity: $2[0,6]$ vs $1[0,6] ; p=0.008)$. Household income, history of preterm delivery, and previous progesterone use were similar between groups.

\section{Perceptions of risk associated with PTL and LBW among Zambian women}

One hundred and thirty (88.4\%) participants perceived PTL as a serious concern for a newborn's health. HIV-uninfected women were significantly more likely to perceive PTL as a serious health concern for infants compared to HIV-infected women $(94.8 \%$ vs $81.4 \%$; RR $=2.39$ [CI $=1.00-5.70]$; $p=0.011)$. Further, 39 (26.5\%) participants were personally worried about having a preterm delivery. HIV-infected respondents were more likely than HIV-uninfected women to be concerned about PTL in their current pregnancy (34.3\% vs $19.5 \% ; \mathrm{RR}=1.45[\mathrm{CI}=1.04-2.01] ; p=0.042)$. Women with previous PTL are not personally more concerned for PTL in their current pregnancy ( $30.6 \%$ vs $25.9 \% ; p=0.746$ ) (Figure 1 ).

General perceptions of the impact of LBW on infant health were not significantly different between HIV-infected and HIV-uninfected women; 113 (76.9\%) women believed that LBW is a serious concern for infant health. When asked about their current pregnancy specifically, 36 (24.5\%) women were worried that their newborn would be born with LBW.

\section{Acceptability of medication to prevent PTL and LBW}

One of the 147 participants did not complete the questions on the acceptability of medications to prevent PTL and LBW. Of the remaining 146 participants, 144 (98.6\%) indicated they would take a medication to prevent PTL and LBW; of note, the two who would not, were HIV-uninfected.

\section{Acceptability of oral formulation of progesterone}

Of the 144 participants willing to take a medication for preventing PTL and LBW, 141 (97.9\%) would take the medication orally. One hundred and forty $(97.2 \%)$ would take the medication daily, and 136 (94.4\%) would take the medication from GW 20 to delivery. There was no difference in the acceptability of oral progesterone between the HIV-infected and uninfected groups ( $p=0.347)$.

\section{Acceptability of intramuscular injections of progesterone}

Intramuscular injections were acceptable to 120 (83.3\%) participants; further, weekly injections were acceptable to 119 (82.6\%). One hundred and eighteen (81.9\%) participants would be willing to receive this intervention in the clinic, while none were willing to administer intramuscular injections at home. HIV-infected women were more likely to accept intramuscular injections compared to HIV-uninfected women $(90.0 \%$ vs $77.0 \% ; \mathrm{RR}=1.800[\mathrm{CI}=0.943-3.435] ; p=0.037)$

\section{Acceptability of vaginal administration of progesterone}

One hundred and twenty-one (84.0\%) participants were willing to use a vaginal formulation. Of these women, $49(34.0 \%)$ were willing to use a vaginal tablet, $58(40.3 \%)$ 
Table I Demographic features of included sample

\begin{tabular}{|c|c|c|c|c|}
\hline Demographic features & $\begin{array}{l}\text { Total participants } \\
(n=147)\end{array}$ & $\begin{array}{l}\text { HIV-infected } \\
(\mathrm{n}=70)\end{array}$ & $\begin{array}{l}\text { HIV-uninfected } \\
(n=77)\end{array}$ & p-value ${ }^{a}$ \\
\hline Age, mean $\pm S D$ & $28.3 \pm 5.7$ & $29.9 \pm 6.04$ & $26.7 \pm 4.98$ & 0.001 \\
\hline $\begin{array}{l}\text { Gestational age at visit } \\
\text { (weeks), mean } \pm \mathrm{SD}\end{array}$ & $\begin{array}{l}36.0 \pm 0.73 \\
(n=130)\end{array}$ & $\begin{array}{l}36.1 \pm 0.70 \\
(n=60)\end{array}$ & $\begin{array}{l}36.0 \pm 0.77 \\
(n=70)\end{array}$ & 0.227 \\
\hline $\begin{array}{l}\text { Monthly household income (ZMW), } \\
\text { mean } \pm \text { SD }\end{array}$ & $\begin{array}{l}\mathrm{I}, 886 \pm 2,528 \\
(\mathrm{n}=135)\end{array}$ & $\begin{array}{l}|, 480 \pm I, 59| \\
(\mathrm{n}=64)\end{array}$ & $\begin{array}{l}2,252 \pm 3,109 \\
(\mathrm{n}=7 \mathrm{I})\end{array}$ & 0.076 \\
\hline Marital status, n (\%) & & & & 0.059 \\
\hline Single & $30(20.4)$ & $18(25.7)$ & $12(15.6)$ & \\
\hline Married & II $3(76.9)$ & $48(68.6)$ & $65(84.4)$ & \\
\hline Divorced & $3(2.0)$ & $3(4.3)$ & $0(0)$ & \\
\hline Windowed & I $(0.7)$ & $\mathrm{I}(\mathrm{I} .4)$ & $0(0)$ & \\
\hline Separated & $0(0)$ & $0(0)$ & $0(0)$ & \\
\hline ^Highest educational level, $\mathrm{n}(\%)$ & & & & 0.010 \\
\hline Less than primary & $28(19.0)$ & $18(25.7)$ & $10(13.0)$ & \\
\hline Primary education & $59(40.1)$ & $32(45.7)$ & $27(35.1)$ & \\
\hline Secondary education & $30(20.4)$ & $7(10.0)$ & $23(29.9)$ & \\
\hline Post-secondary education & $29(19.7)$ & $13(18.6)$ & $16(20.8)$ & \\
\hline Gravidity (number of pregnancies), n (\%) & & & & 0.015 \\
\hline 1 & $40(27.2)$ & $16(22.9)$ & $24(31.2)$ & \\
\hline 2 & $33(22.4)$ & II (I5.7) & $22(28.6)$ & \\
\hline 3 & $30(20.4)$ & $16(22.9)$ & $14(18.2)$ & \\
\hline 4 & $27(18.4)$ & I5 (21.4) & $12(15.6)$ & \\
\hline$\geq 5$ & $17(\mid I .5)$ & $12(17.1)$ & $5(6.5)$ & \\
\hline Parity (number of deliveries), $\mathrm{n}(\%)$ & & & & 0.006 \\
\hline 0 & $43(29.3)$ & $17(24.3)$ & $26(33.8)$ & \\
\hline 1 & $36(24.5)$ & $12(17.1)$ & $24(31.2)$ & \\
\hline 2 & $28(19.0)$ & $15(21.4)$ & $13(16.9)$ & \\
\hline 3 & $26(17.7)$ & $16(22.9)$ & $10(13.0)$ & \\
\hline$\geq 4$ & $14(9.6)$ & $10(14.2)$ & $4(5.2)$ & \\
\hline History of previous preterm births, $n(\%)$ & & & & 0.730 \\
\hline Yes & $13(8.9)$ & $6(8.6)$ & $7(9.1)$ & \\
\hline No & $134(9 \mid .2)$ & $64(91.4)$ & $70(90.9)$ & \\
\hline Previous use of progesterone, $\mathrm{n}(\%)$ & & & & 0.623 \\
\hline No previous use & $64(43.5)$ & $29(4 I .4)$ & $35(45.5)$ & \\
\hline Yes previous use & $83(56.5)$ & $41(58.6)$ & $42(54.5)$ & \\
\hline \multicolumn{5}{|l|}{ Mode of administration of previous } \\
\hline progesterone, $\mathrm{n}(\%)$ & & & & \\
\hline Oral progesterone ${ }^{\mathrm{b}}$ & $38(25.9)$ & $19(24.7)$ & $19(27.1)$ & 0.931 \\
\hline Intramuscular progesterone $\mathrm{b}^{\mathrm{b}}$ & $54(36.7)$ & $24(34.3)$ & $30(39.0)$ & 0.210 \\
\hline Implanted contraceptive device ${ }^{b}$ & $7(4.8)$ & $5(7.1)$ & $2(2.6)$ & 0.336 \\
\hline
\end{tabular}

were willing to use a vaginal gel, and 14 (9.7\%) were willing to use either formulations. One hundred and eighteen participants $(81.9 \%)$ were willing to use the medication (tablet or gel) daily, and 120 participants $(83.3 \%)$ were willing to use the vaginal medication from GW 20 to delivery. There was a trend toward higher acceptability of vaginal administration in HIV-infected compared to HIV-uninfected women $(90.0 \%$ vs 78.4\%; RR $=1.711[\mathrm{CI}=0.901-3.248] ; p=0.057$ ).

The acceptability of the surveyed modes of medication administration is summarized in Figure 2.

\section{Overall preference between intramuscular injections, vaginal tablets, and vaginal gels}

Choosing between the currently recommended formulations to prevent PTL in at-risk women, 89 of 147 participants $(60.5 \%)$ preferred intramuscular administration, $22(15.0 \%)$ preferred vaginal tablets, and $36(24.5 \%)$ preferred vaginal gels (Figure 3). There was no difference in preference between HIV-infected and HIV-uninfected women $(p=0.421)$. 


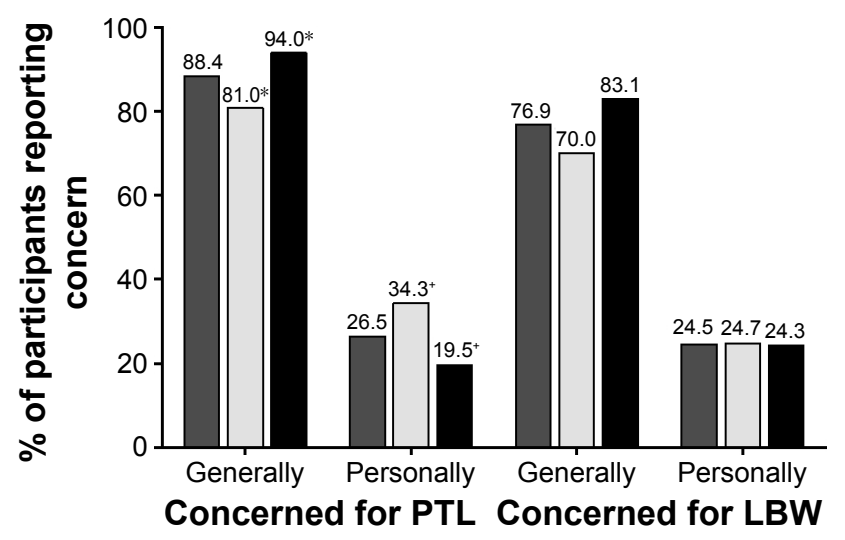

$\square$ All participants (n=147) $\square$ HIV-infected (n=70) - HIV-uninfected $(\mathrm{n}=77)$

Figure I General and personal concerns for preterm labor (PTL) and low birth weight (LBW) in HIV-infected and HIV-uninfected women.

Note: *and +indicate a significant difference $(p<0.05$, chi squared) between HIVinfected and HIV-uninfected women.

\section{Potential factors affecting progesterone supplementation acceptance}

Participants who had previously used intramuscular progesterone were more likely to prefer intramuscular delivery of progesterone compared to women with no previous experience with intramuscular delivery $(74.1 \%[40 / 54]$ vs $52.7 \%$ [48/91]; RR: 1.862 [CI $=1.117-3.104 ; p=0.011]$ ). Though not reaching significance $(p=1.000)$, all women with previous preterm deliveries accepted either intramuscular or vaginal medication to prevent PTL and LBW, as opposed to

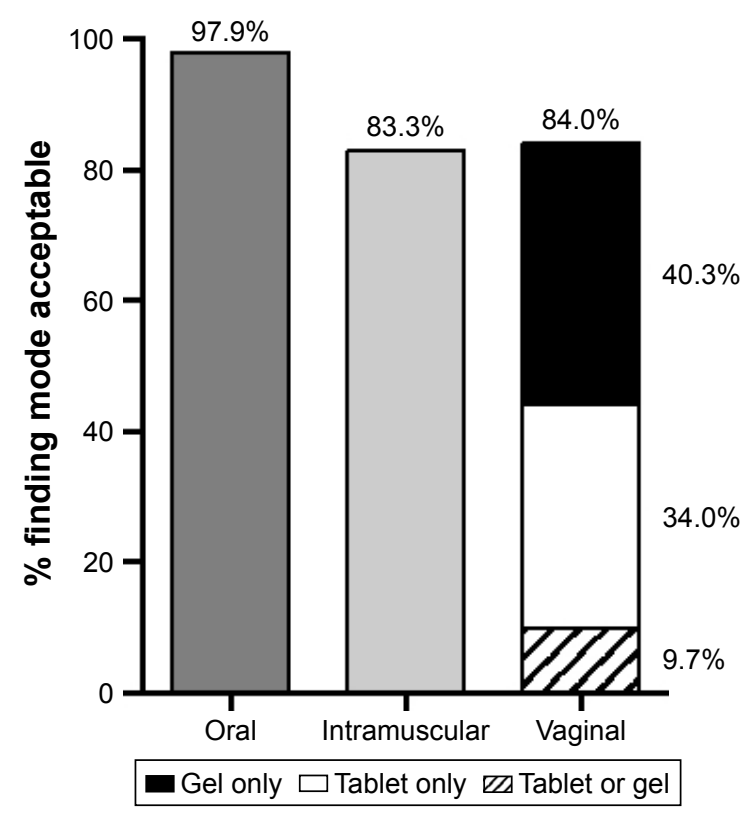

Figure 2 Acceptability of various modes of medication delivery for the prevention of preterm labor and low birth weight.

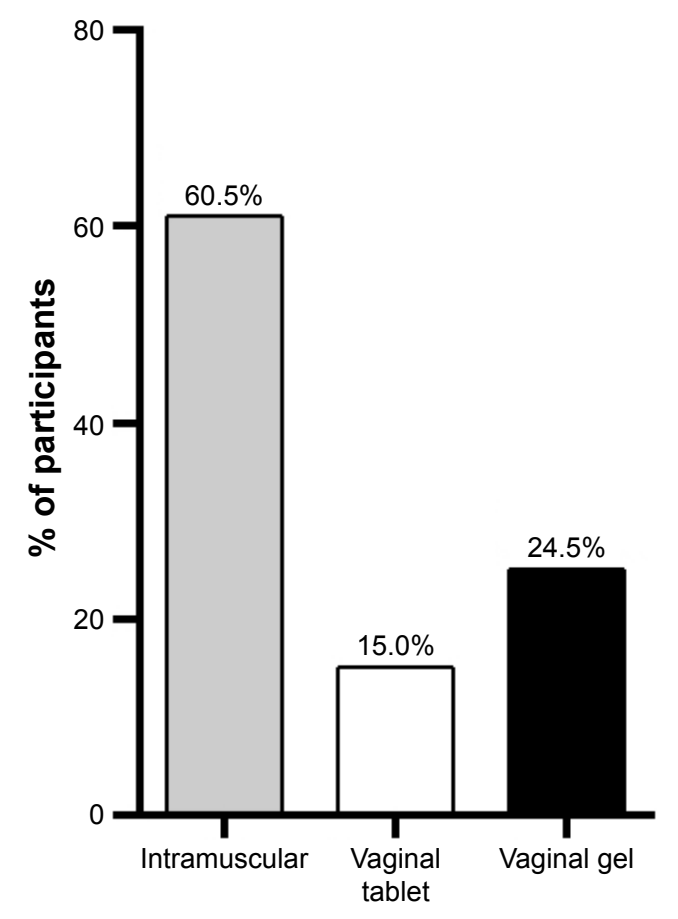

Figure 3 Participant preference between the currently recommended modes of progesterone supplementation for the prevention of preterm labor and low birth weight $(n=144)$.

93.3\% of women with no previous experience with preterm deliveries. HIV-infected ( $\mathrm{n}=70)$ and HIV-uninfected women $(n=77)$ demonstrated a similar preference for intramuscular or vaginal medication in the prevention of PTL and LBW (65.8\% [46/70] vs 55.8\% [43/77]; $p=0.221)$.

Overall, 138 of 146 (94.5\%) women would be willing to take either an intramuscularly or vaginally delivered medication to prevent PTL and LBW. Only eight women $(5.5 \%)$ would not consider taking a medication or would only consider an oral medication to prevent PTL/LBW.

\section{Discussion}

To our knowledge, this was the largest study to date addressing the attitudes of women in low resource settings toward progesterone supplementation for prevention of PTL and LBW. The results of this study suggest that progesterone supplementation for the prevention of PTL and LBW can be considered an acceptable option in Zambian women. There is a high degree of acceptability (94.5\% of participants) for intramuscular or vaginal administrations of progesterone among HIV-infected and HIV-uninfected women. This supports the implementation of ACOG- and SOGC-indications for supplemental progesterone in Zambia. ${ }^{8,9}$ If the efficacy of progesterone supplementation is demonstrated in cART-associated PTL and LBW, then these results support 
supplemental intramuscular or vaginal progesterone as an acceptable intervention for HIV-infected Zambian women.

Of the approved delivery modes (ie, excluding oral administration), this study demonstrated a preference for intramuscular administration over vaginal preparations of progesterone. This study contrasts with previous reports ${ }^{16,17}$ that demonstrated a preference for vaginal administration, one of which included only four mothers and so may not have been representative. ${ }^{17}$ In an American study completed post-intervention on in vitro fertilization, patients reported higher acceptability for vaginal progesterone due to less discomfort from vaginal administration compared to intramuscular injections. ${ }^{16,17}$ Since our study was completed prior to intervention, this difference may be attributable to familiarity with intramuscular medications in Zambia. This familiarity hypothesis is supported by a significantly greater preference for intramuscular progesterone over vaginal progesterone in individuals with previous exposure to intramuscular progesterone (eg, Depo-Provera ${ }^{\circledR}$ ) in our study. Another explanation for the discrepancy may be due to differences in cultural norms and gender roles in developing countries that may affect the uptake of vaginal medications. ${ }^{18}$

This study suggests that providing options may improve acceptability of the rollout of a progesterone supplementation program given that $94.5 \%$ of women would accept intramuscular or vaginal progesterone. Acceptability decreases to $83 \%-84 \%$ if given only one of the choices. Since there is no superiority demonstrated between vaginal or intramuscular progesterone to prevent PTL, this remains a personal choice for patients.

The acceptability of progesterone supplementation may be driven by a strong perception that PTL and LBW seriously affect infant health. However, fewer women were personally concerned about PTL and LBW. Interestingly, women with a history of PTL (ie, women at high risk for PTL) are not more willing to take medication to prevent PTL and LBW. This knowledge gap regarding risk factors for PTL and LBW has been demonstrated in interviews with patients, community health workers, and clinical officers in Malawi. ${ }^{17}$ However, due to the limited number of women with previous preterm deliveries ( $n=13)$, our study may not be powered to detect a small difference. Despite similar experience with previous PTL, HIV-infected participants were more concerned about PTL for their current pregnancy compared to HIV-uninfected women. This may be because HIV-infected women inherently believe their infection predisposes them to PTL or perhaps, they have been previously counseled regarding their increased risk.

\section{Limitation}

There was a high level of attrition in this study (14.4\% and $48.5 \%$ for the HIV-infected and HIV-uninfected cohorts, respectively). This may be because the study follow-up visit and routine antenatal visits were not coordinated. Retention in the HIV-infected group may be higher because they attend the same clinic for their HIV care, allowing for more opportunities to engage in the study. Furthermore, the closure of the obstetrics ward at the clinic during the study period redirected pregnant women away from the clinic for their deliveries. The loss of participants unable to manage the extra trip to the clinic means the perceptions of women with limited resources may be missed.

All study participants were enrolled in a study to assess normal progesterone levels in pregnant Zambian women, and their attitudes to progesterone supplementation may not reflect those of the general population. However, certain groups of women who have demonstrated willingness for progesterone use (currently using progesterone) or at increased risk of PTL/LBW (currently using PI-based cART) ${ }^{12,13}$ were excluded from the study, and so we do not think that the acceptability was over-estimated. In addition, since this was a non-interventional study (which allowed women who would as well as those who would not accept treatment to enroll), the acceptability of progesterone supplementation to prevent these complications should reflect the attitudes in the general community of pregnant women.

This study may influence the provision of supplemental progesterone in Zambia and other resource-limited settings. While considered acceptable to HIV-infected women, the efficacy of supplemental progesterone to prevent PTL and $\mathrm{LBW}$ in this population remains to be determined. A pilot study of progesterone supplementation in HIV-infected pregnant women is currently underway in Canada. ${ }^{19}$ At a health systems level, there is a need for cost-benefit analysis of progesterone supplementation in preventing PTL and LBW, and cost-effectiveness analysis of modes of administration.

\section{Conclusion}

PTL and LBW are perceived to be serious concerns for infant health among both HIV-infected and HIV-uninfected Zambian women. Over $95 \%$ of women surveyed would consider taking medications to prevent PTL and LBW. Oral, intramuscular, and vaginal modes of administration were considered acceptable to the women, with the oral administration being preferred. Between the approved modes of progesterone administration for preventing PTL and LBW, intramuscular administration was preferred, likely due to 
previous experience with this mode. These data could inform the successful implementation of a progesterone supplementation program to prevent PTL and LBW in Zambia and other similar settings.

\section{Acknowledgments}

The study was supported by funding from the University of Toronto Medical Alumni Association (JMHW, ND, and NK) and the Canadian Institutes of Health Research (CIHR) grant number MOP-130398 (LS and MS).

This research was presented at the American College of Obstetricians and Gynecologists' 2017 Annual Clinical and Scientific Meeting, held at San Diego, California, May 6-9, 2017 , as a poster with interim findings. The abstract was published in Obstetrics \& Gynecology, May 2017: Volume 126; Supplement 1. NK and ND were co-second authors for this work. LS and MS were co-senior authors for this work.

\section{Author contributions}

JMHW contributed to the planning, data collection, data analysis, and manuscript writing. NK and ND contributed to the data collection, data analysis, and manuscript writing. MN contributed to the design of the study and the manuscript editing. LS contributed to the design, data analysis of the study, and the manuscript editing. MS contributed to the design of the study, and manuscript editing. All authors contributed toward data analysis, drafting and revising the paper and agree to be accountable for all aspects of the work.

\section{Disclosure}

The authors report no conflicts of interest in this work.

\section{References}

1. Blencowe H, Cousens S, Chou D, et al. Born too soon: the global epidemiology of 15 million preterm births. Reprod Health. 2013; 10 (Suppl 1):S2.

2. Blencowe H, Cousens S, Oestergaard MZ, et al. National, regional, and worldwide estimates of preterm birth rates in the year 2010 with time trends since 1990 for selected countries: a systematic analysis and implications. Lancet. 2012;379(9832):2162-2172.

3. Petrou S, Mehta Z, Hockley C, Cook-Mozaffari P, Henderson J, Goldacre $\mathrm{M}$. The impact of preterm birth on hospital inpatient admissions and costs during the first 5 years of life. Pediatrics. 2003;112(6 Pt 1): 1290-1297.
4. Goldenberg RL, Culhane JF, Iams JD, Romero R. Epidemiology and causes of preterm birth. Lancet. 2008;371(9606):75-84.

5. Muglia LJ, Katz M. The enigma of spontaneous preterm birth. $N$ Engl J Med. 2010;362(6):529-535.

6. Mackenzie R, Walker M, Armson A, Hannah ME. Progesterone for the prevention of preterm birth among women at increased risk: a systematic review and meta-analysis of randomized controlled trials. Am J Obstet Gynecol. 2006;194(5):1234-1242.

7. Dodd JM, Jones L, Flenady V, Cincotta R, Crowther CA. Prenatal administration of progesterone for preventing preterm birth in women considered to be at risk of preterm birth. Cochrane Database Syst Rev. 2013;(7):CD004947.

8. Society for Maternal Fetal Medicine Publications Committee. ACOG Committee Opinion number 419 October 2008 (replaces no. 291, November 2003). Use of progesterone to reduce preterm birth. Obstet Gynecol. 2008;112(4):963-965.

9. NorwitzER, Caughey AB. Progesterone supplementation and the prevention of preterm birth. Rev Obstet Gynecol. 2011 Summer;4(2):60-72.

10. Townsend C, Schulte J, Thorne C, et al. Antiretroviral therapy and preterm delivery-a pooled analysis of data from the United States and Europe. BJOG. 2010;117(11):1399-1410.

11. Kourtis AP, Schmid CH, Jamieson DJ, Lau J. Use of antiretroviral therapy in pregnant HIV-infected women and the risk of premature delivery: a meta-analysis. AIDS. 2007;21(5):607-615.

12. Powis KM, Kitch D, Ogwu A, et al. Increased risk of preterm delivery among HIV-infected women randomized to protease versus nucleoside reverse transcriptase inhibitor-based HAART during pregnancy. $J$ Infect Dis. 2011;204(4):506-514.

13. Papp E, Mohammadi H, Loutfy MR, et al. HIV protease inhibitor use during pregnancy is associated with decreased progesterone levels, suggesting a potential mechanism contributing to fetal growth restriction. J Infect Dis. 2015;211(1):10-18.

14. RamaRao S, Clark H, Rajamani D, et al. Progesterone vaginal ring: results of a three-country acceptability study. New York, Population Council. 2015:33.

15. Wieder DR, Pattimakiel L. Examining the efficacy, safety, and patient acceptability of the combined contraceptive vaginal ring (NuvaRing). Int $J$ Womens Health. 2010;2:401-409.

16. Yanushpolsky E, Hurwitz S, Greenberg L, Racowsky C, Hornstein M. Crinone vaginal gel is equally effective and better tolerated than intramuscular progesterone for luteal phase support in in vitro fertilizationembryo transfer cycles: a prospective randomized study. Fertil Steril. 2010;94(7):2596-2599.

17. Levison J, Nanthuru D, Chiudzu G, Kazembe PN, Phiri H, Ramin SM, Aagaard KM. Qualitative assessment of attitudes and knowledge on preterm birth in Malawi and within country framework of care. BMC Pregnancy Childbirth. 2014;14:123.

18. Justin-Temu M, Damian F, Kinget R, Van Den Mooter G. Intravaginal gels as drug delivery systems. $J$ Womens Health (Larchmt). 2004;13(7):834-844.

19. Siou K, Walmsley SL, Murphy KE, et al. Progesterone supplementation for HIV-positive pregnant women on protease inhibitor-based antiretroviral regimens (the ProSPAR study): a study protocol for a pilot randomized controlled trial. Pilot Feasibility Stud. 2016;2:49.
International Journal of Women's Health

\section{Publish your work in this journal}

The International Journal of Women's Health is an international, peerreviewed open-access journal publishing original research, reports, editorials, reviews and commentaries on all aspects of women's healthcare including gynecology, obstetrics, and breast cancer. The manuscript management system is completely online and includes

\section{Dovepress}

a very quick and fair peer-review system, which is all easy to use. Visit http://www.dovepress.com/testimonials.php to read real quotes from published authors. 